\title{
Modelo para la formación de profesores en y para la diversidad
}

Recibido: 15 Ago 2016 - Revisado: 30 Sep 2016 Aceptado: 30 Oct 2016 - Publicado: 30 Dic 2016

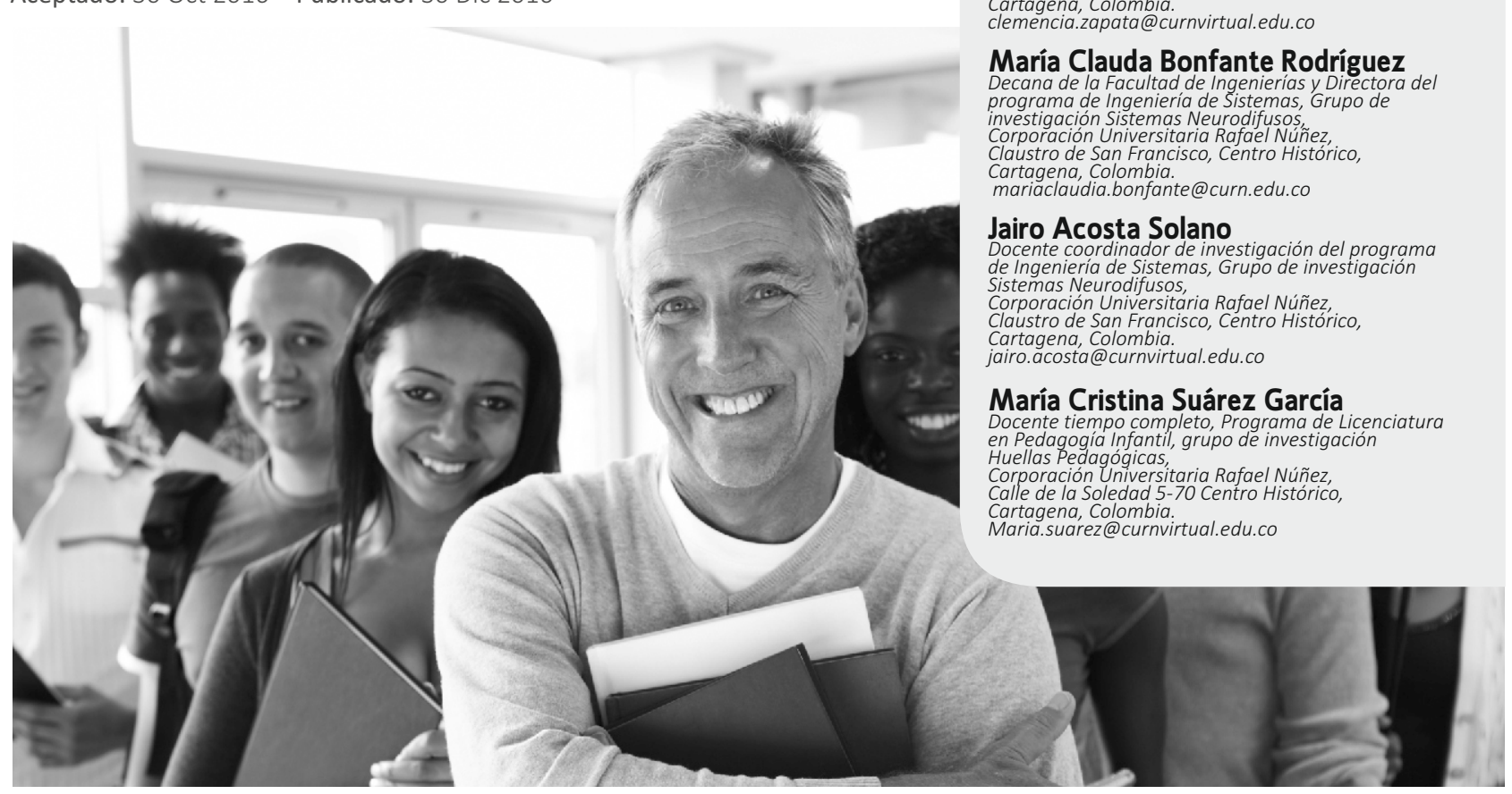

Resumen: Este trabajo presenta la dinámica de la construcción del modelo de formación del profesorado en y para la diversidad, un proyecto de investigación que pertenece al programa nacional "Arquitectura pedagógica, didáctica y tecnológica para formación de profesores en y para la diversidad", financiado por Colciencias, en el contrato 546-2014, donde se explica la composición, funciones, diseño de protocolos, producción de contenidos digitales educativos y las plantillas de diseño de instrucción que dirigen el proceso de enseñanza y aprendizaje. La metodología de diseño aplicado fue desarrollada en fases: documental, de diagnóstico, epistemológica, marco teórico y metodológico, diseño, producción, validación y difusión. Se destaca, como producto nuclear, el campus virtual "Apropia-dos", desplegado en tres plataformas con recursos digitales, "Tutoría-dos", "Deposita-dos" y "Utiliza-dos", en las cuales se encuentran, respectivamente, los cursos de formación del profesorado, los recursos educativos digitales producidos y los utilizados.

Palabras clave: Modelo de formación; profesores; enseñanza; aprendizaje; sordos; contenidos educativos digitales.

Abstract: This paper presents the dynamic model building of teacher training and diversity, a research project belonging to the national program "teaching architecture, teaching and technology for teacher training in and for diversity" financed by COLCIENCIAS, the contract 546-2014, explaining the composition, functions, protocol design, production of educational digital content and instructional design templates that direct the process of teaching and learning. The design methodology applied was developed in phases: documentary, diagnostic, epistemological, theoretical and methodological framework, design, production, validation and dissemination. Stands out as the core product, the virtual campus "Grabber-dos", deployed in three platforms with digital resources, "Tutoria-dos," "Deposita-dos" and "Utiliza-dos" in which they are, respectively, the training of teachers, educational resources produced and used.

Key words: Training model, teachers, teaching, learning, deaf, digital educational content. 


\section{INTRODUCCIÓN}

En el marco del programa Nacional "Arquitectura pedagógica, didáctica y tecnológica para formación de profesores en y para la diversidad", financiado por COLCIENCIAS bajo contrato 546-2014, se desarrolla en la Corporación Universitaria Rafael Núñez, el proyecto "Modelo para producción de contenido digital educativo para inclusión de sordos", una sinergia lograda entre Pedagogía e Ingeniería; sustentando la gestión del proyecto en el trabajo integral desarrollado por comunidades de práctica (Wenger 2001), las cuales están desplegadas en: pedagógica, tecnológica, didáctica y de gestión de conocimiento.

Esta investigación coadyuva con la intencionalidad de la Política Nacional propuesta en "Paz, equidad y Educación" que es acogida por Ciencia, Tecnología e Innovación; entendiendo la diversidad como una dinámica social que articula procesos de reconocimiento de promoción, cooperación y crecimiento con lo otro, con los otros y con sus diferencias, desde esta perspectiva, el sistema educativo reconoce el derecho a la igualdad (Universidad Internacional de Andalucía 2008), al acceso de formas de explorar, construir y comunicar conocimiento con todos y para todos; valorando la diversidad como una riqueza propia de la humanidad. En el anterior sentido, se propone "desarrollar modelos para la apropiación y el uso de tecnologías en escenarios educativos que acogen diversidad de poblaciones", atendiendo la siguiente ruta: (i). identificar y determinar el enfoque epistemológico, teórico y metodológico del modelo a partir de una línea base que caracterice al educando sordo, los estándares curriculares, los estilos y objetivos de aprendizaje; (ii) diseñar un modelo instruccional para la apropiación y uso de TIC que permita potenciar los procesos pedagógicos que medien la inclusión de sordos en contextos educativos. (iii) Implementar el modelo desplegando la producción y usabilidad de recursos digitales para sordos como apoyo a las mediaciones didácticas propuestas y desarrolladas por los docentes; y (iv) valorar el impacto pedagógico y didáctico del modelo y su potencial de apropiación y uso en las escuelas con sordos.

Con estas premisas, la metodología desplegada para la realización de este proyecto, da cuenta de una investigación aplicada, que incluye una investigación documental y diagnóstica, desplegada en fases: (i) encuadre epistemológico, teórico y metodológico; (ii) caracterización de aprendizajes; (iii) diseño del modelo con estrategias Pedagógicas y Tecnológicas; (iv) desarrollo e implementación del modelo; (v) valoración de impacto; (vi) divulgación.

Se inicia la consolidación de la línea base por los grupos de investigación Sistemas Neurodifusos y Huellas Pedagógicas de la Corporación Universitaria Rafael Núñez, caracterizando las instituciones educativas, docentes y población sorda, para identificar:

- La infraestructura tecnológica de las instituciones y el nivel de conocimiento en Tecnologías de la Información y Comunicación (TIC) de docentes de sordos.

- Los estándares y objetivos de aprendizaje para la población sorda.

- Las teorías pedagógicas y metodológicas vigentes en contextos inclusivos.

- Los modelos pedagógicos de las instituciones educativas que atienden población sorda.

- Los estilos de aprendizaje de la población sorda.

- Aspectos administrativos, recursos educativos y políticas existentes en las escuelas que atienden sordos.

Lo anterior, develó el bajo nivel de apropiación de los conceptos de entornos y objetos virtuales de aprendizaje, tanto en profesores como en estudiantes, limitaciones en cuanto al uso de recursos didácticos que motiven y potencien el aprendizaje de 
sordos en estos contextos educativos inclusivos; deficiencias en infraestructura y recursos tecnológicos; heterogeneidad marcada en cuanto a diagnóstico y edad de los estudiantes sordos; insuficiencia en cuanto a adecuaciones concretas en los planes de estudio para sordos; pocas investigaciones, en particular ninguna sobre estilos cognitivos de la población en condición de discapacidad auditiva.

\section{MODELO PARA PRODUCCIÓN DE CONTENIDO DIGITAL EDUCATIVO}

Esta propuesta surge con la emergencia de la necesidad, en cuanto a formación de profesores y de mejora en los aprendizajes de los sordos, preocupación manifiesta y confirmada por Instituto Nacional para Sordos (INSOR), en las pruebas SABER, donde la población estudiantil sorda está por debajo de la estadística más baja de desempeño en estudiantes regulares.

Con estos antecedentes se diseña y construye el «Modelo para producción de contenido digital» (ver Fig. 1), definiendo el carácter del modelo, en lo formativo, desde la pragmática de formar profesores para mejorar su desempeño laboral en escenarios de enseñanza y de aprendizaje y en competencias TIC, bajo la perspectiva del Ministerio de Educación y de la asunción de la Fundación telefónica (2012) donde afirma que el soporte tecnológico es indispensable en los procesos de formación.

En lo didáctico desde la perspectiva de considerarlo un instrumento de análisis, intervención y mejora de la práctica educativa, basado en el desarrollo de metodologías de "investigación escolar", de trabajo en torno a "problemas", con secuencia de actividades relativas al tratamiento de esos problemas, donde el profesor es coordinador de los procesos porque funge como "investigador en el aula" (García 2000); e Innovador, donde se propende por una "cultura innovadora" en la cual los docentes innovadores se autorreconocen como tales, están abiertos a transformar su práctica, se arriesgan, planifican y son novedosos en sus estrategias de cambio, trabajando en equipo hacia una meta común (UNESCO 2006).

El modelo (Fig. 1) se estructura desde el principio de Incertidumbre (Heisenberg 1985), pues los cambios en el mundo son vertiginosos e impactan en los sistemas educativos, esta incertidumbre, avoca a las comunidades a mejorar la calidad de la educación, en el marco de la libertad pedagógica, para formar mejores educadores.

Por otro lado, el acercamiento a la incertidumbre lo hace Morin (1993) planteando «Hay que aprender a enfrentar la incertidumbre puesto que vivimos una época cambiante donde los valores son ambivalentes, donde todo está ligado. Es por eso que la educación del futuro debe volver sobre las incertidumbres ligadas al conocimiento» y, Bárcena (2005) quien propone el tratamiento de la incertidumbre en la enseñanza reflexiva. Otro elemento teórico de soporte, es el paradigma de la complejidad, asumido por Morín (2005), quien avoca a cambios sustanciales en el conocimiento de las ciencias, la educación, la cultura y la sociedad.

También, el modelo se fundamenta en la propuesta de Begoña Vitoriano (2007), quien propone la teoría de la decisión, decisión con incertidumbre o riesgo y una teoría de juegos, que bien se enmarcan en las posibilidades del contexto educativo y de los sujetos que en el interactúan 


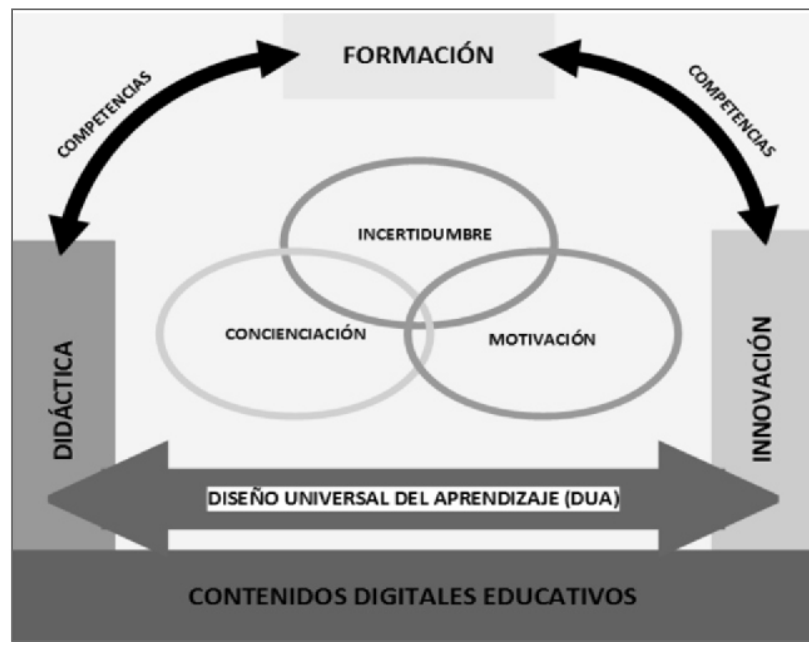

Figura 1. Modelo para producción de contenido digital.

Desde otros ángulos, el modelo pone en juego el fenómeno de la concienciación, tomando aspectos planteados por Kriegel (2009), relacionados con la conciencia fenoménica y las posturas ficisistas y antifisicistas, que se revelan en los contextos formativos, dejando a discreción en el modelo, los planteamientos teóricos sobre la motivación en el proceso de enseñanza y de aprendizaje; otros sobre la búsqueda de intereses, con las opciones de mantenimiento del esfuerzo, de persistencia y de autorregulación.

Se incorporan en el modelo, los principios y objetivos del Diseño Universal de Aprendizaje (2006), en un intento por visibilizar la inclusión de lo diverso y de validar la necesidad de generar recursos y ambientes educativos abiertos y accesibles, y se incluye el sistema de competencias TIC recomendadas por el MEN (2013), para el desarrollo profesional docente: tecnológica, pedagógica, comunicativa, de gestión e investigativa.

Roles: para el desarrollo del modelo se determinó una estructura de niveles con roles como dirección, asesoría y soporte (ver Fig. 2).

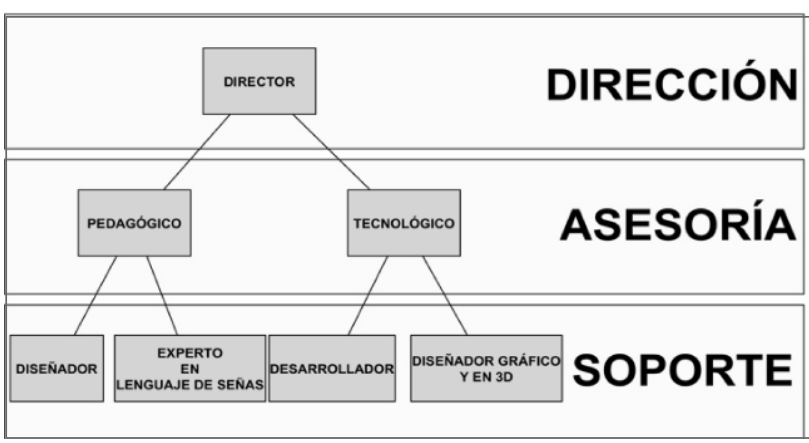

Figura 2. Roles y responsabilidades.

El modelo para la producción de contenido digital educativo se despliega en cuatro componentes (ver Figura 3), que representan los subsistemas del sistema global, los mecanismos de integración, las posibles interacciones y dependencias entre ellos y las tecnologías requeridas para su soporte.

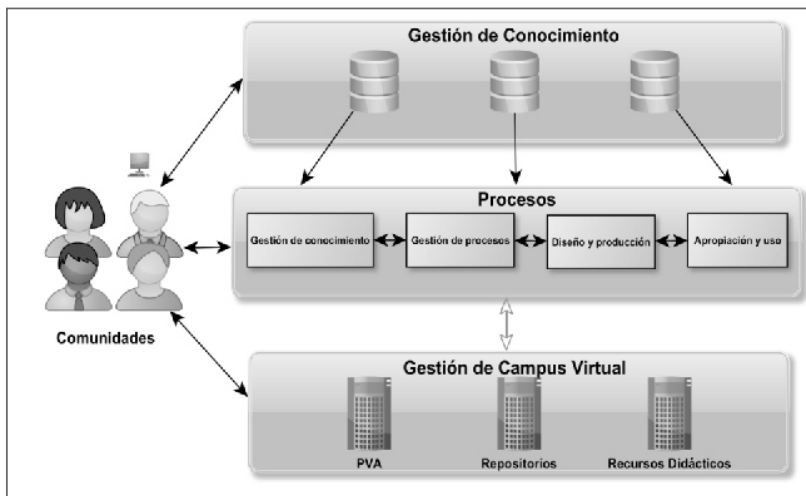

Figura 3. Componentes del modelo de producción de contenidos digitales educativos.

La pragmática del modelo para producción de contenidos digitales, invita a la creación de comunidades de práctica, esto implica la validación del modelo per sé y de las comunidades generadas, lo que se soportará y validará en la teoría de conformación de redes, mediante los modelos de Gran Componente, de Acoplamiento Preferencial y el Viral.

(a) Comunidades de práctica: los docentes y directivos en sus prácticas tienen la responsabilidad de incluir a 
los estudiantes, asumiendo su condición de diversidad manifiesta o no, como una oportunidad para dignificarlos en el marco de una cultura para y por la paz, de hacer gala del principio de equidad para mejorar con ello los aprendizajes y calidad de la educación.

El modelo prevé la conformación de diversas comunidades desplegadas en un abanico disciplinar: una con intereses didácticos, otra integrada por entidades gubernamentales; de otro lado, se espera la emergencia de la comunidad de sordos integrada por profesores sordos, sordos autónomos, modelos lingüísticos, padres de sordos y comunidad educativa, alrededor de la población sorda.

(B) Gestión de Conocimiento: este componente mantendrá el conocimiento de las comunidades de práctica (Wenger 2001), haciendo visibles los intereses particulares del mismo en estos grupos, su condición, su nivel de uso de TIC, nuevas necesidades de formación y motivación frente al uso de otros componentes del modelo, este sistema permitirá retro y realimentarlo.

Investigaciones adelantadas en países angloparlantes encuentran como factor importante, aprovechar las TIC para el aprendizaje de los estudiantes, para mejorar la competencia en TIC del profesor de área e influir en sus creencias, expectativas, flexibilidad al cambio y prácticas pedagógicas.

Es razonable pensar que estos factores pueden tener el mismo peso en Iberoamérica, lo cual se pretende validar con el levantamiento de una línea base, así, este componente de gestión de conocimiento integrará en una base de datos información que permitirá realizar análisis, identificar requerimientos de contenidos y productos, validar los productos y verificar la apropiación de TIC en las comunidades de práctica.

(C) Gestión de procesos: este componente mantiene la lógica en la que se desarrollan los procesos didácticos, pedagógicos, curriculares, de diseño y producción de contenidos contextuados, de validación de contenidos, de uso y apropiación de TIC en las comunidades de práctica. En estos procesos se documentará e identificará a los participantes, las actividades realizadas, la ruta para la toma de decisiones, el soporte normativo, instructivos y otros procedimientos necesarios.

(D) Proceso diseño y producción de contenidos (ver Figura 4): los docentes deben manejar las nuevas y emergentes tecnologías como soporte, para producir recursos educativos y didácticos como: Objetos Virtuales de Aprendizaje (OVA), Objetos Virtuales de Información, repositorios, Web 2.0, videojuegos, libros digitales y otros, soportados en un diseño instruccional.

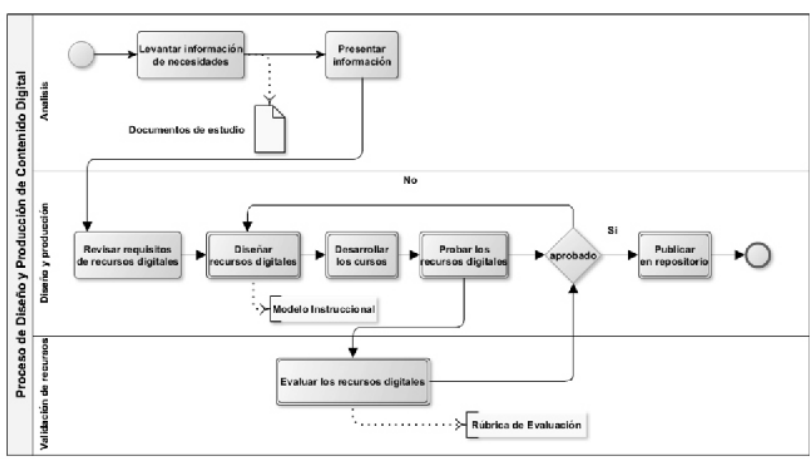

Figura 4. Proceso diseño y producción de contenido digital.

(E) Proceso de apropiación y usabilidad de las TIC: de acuerdo con las directrices emanadas del MEN, un docente en su desarrollo profesional debe integrar efectivamente las TIC en sus procesos de enseñanza/aprendizaje, atendiendo cinco aspectos fundamentales (ver Figura.5): (i) adquirir competencia básica en uso de las TIC; (ii) disponer de estrategias pedagógicas adecuadas que le permitan utilizar el potencial transformador de las TIC para mejorar el aprendizaje de sus estudiantes y (iii) desarrollar competencia para llevar a cabo, cada vez mejor, el proceso de integración efectiva de las TIC en 
su disciplina; (iv) generar competencias investigativas, para buscar la forma de innovar en el aula de clase, (v) desplegar competencias de gestión para la consecución de recursos TIC.

Estos aspectos permitirán reconocer su nivel de clasificación en el uso pedagógico de las TIC como explorador, integrador y/o innovador.

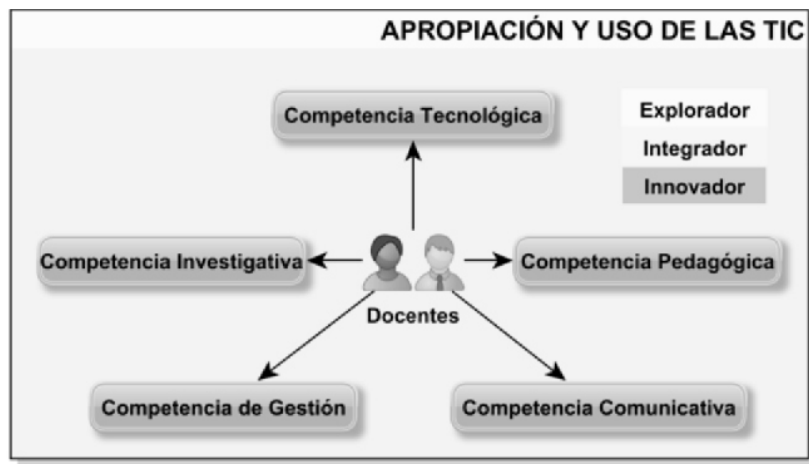

Figura 5. Desarrollo profesional docente.

(F) Gestión del Campus virtual (ver Figura.6): el campus virtual es el componente nuclear que provee una interfaz web para el acceso de las comunidades de práctica a las plataformas virtuales de aprendizaje; en él, se encuentran los repositorios con los cursos, recursos y contenidos educativos digitales, que potenciarán y facilitarán los procesos de enseñanza y aprendizaje de población diversa.

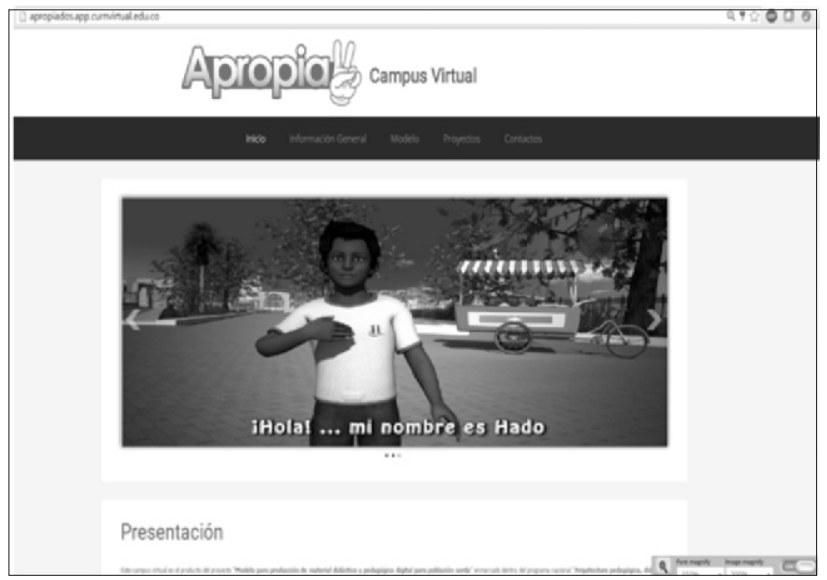

Figura 6. Interface campus virtual.
Para esos efectos, en las plataformas se dispone de tres repositorios (ver Figura 7), que cuentan con un avatar HADO (Hypo Acoustic Doctrinae Obiectum), que se comunica en lengua de señas colombianas y cumple rol de maestro en algunos objetos virtuales de aprendizaje.

Además, dependiendo de los contenidos y las necesidades de accesibilidad de la población estudiantil, se está incluyendo el sistema closed caption, subtitulación, recuadros con modelos lingüísticos y, se cuenta con dispositivos para mejorar la accesibilidad visual para facilitar el trabajo de personas con baja visión.

El campus virtual se habilitará cumpliendo con las pautas de accesibilidad web para atención de la diversidad poblacional.

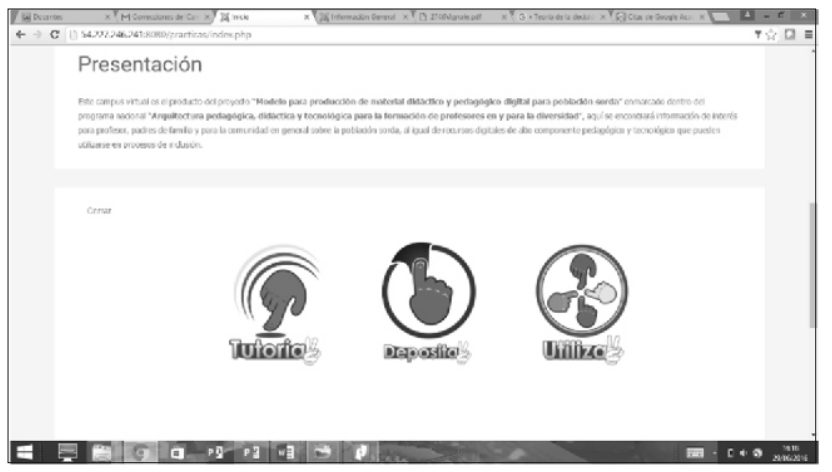

Figura 7. Plataformas con recursos digitales educativos.

En la Fig. 7, se aprecia la plataforma "Tutoria-dos", donde se encuentran diversos cursos, que le permiten a los miembros de comunidades de práctica, acceder a estos, aprender (entre algunos, a diseñar y desarrollar diversos contenidos) y hallar recursos digitales de aprendizaje (ver Figura 8); para depositarlos en la plataforma "Deposita-dos" donde se tendrá la opción de emplear los recursos accediendo a la plataforma "Utiliza-dos". 
Los cursos se encuentran soportados en A-Tutor, ahora se está construyendo la versión de acceso libre para Moodle.

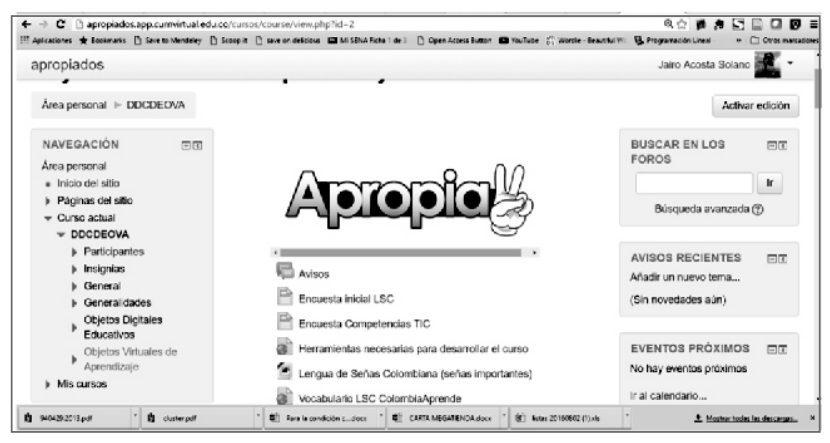

Figura 8. Interfaz de curso para diseño y desarrollo de OVA.

(F) Diseño instruccional: como proceso sistémico, planificado y estructurado, permite producir contenidos digitales para educación tanto presencial como virtual; indica además, cómo llevar a cabo la producción de cursos para la formación en esas modalidades.

Se puede presentar en módulos o unidades didácticas, objetos de aprendizaje y en general recursos educativos, para recrear escenarios de aprendizaje (Nesbit, Belfer y Leacock 2013).

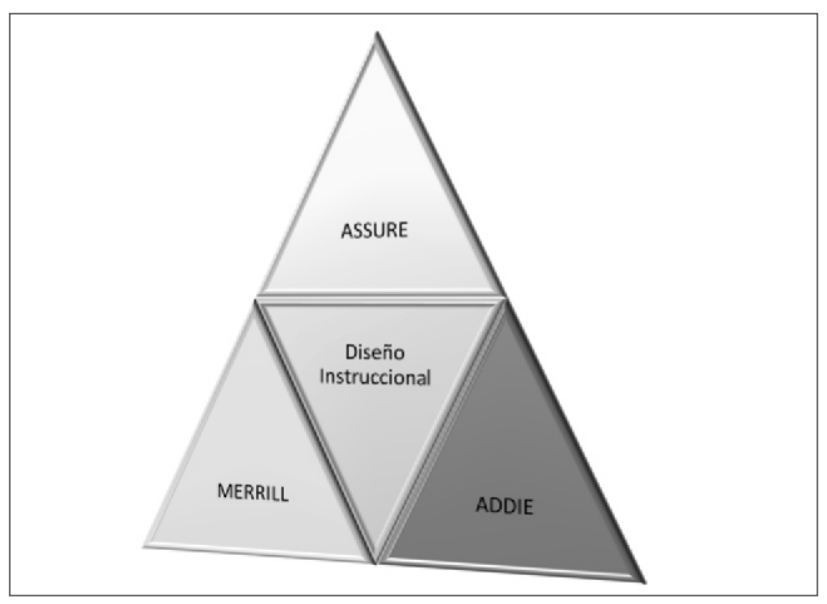

Figura 9. Revisión de diseños instruccionales.
En el proceso, se revisaron diversos diseños instruccionales como se muestra en la Figura 9, recuperando de cada uno elementos que permitieran diseñar la plantilla para el diseño de contenidos y recursos educativos digitales que se muestra en la Figura 10.

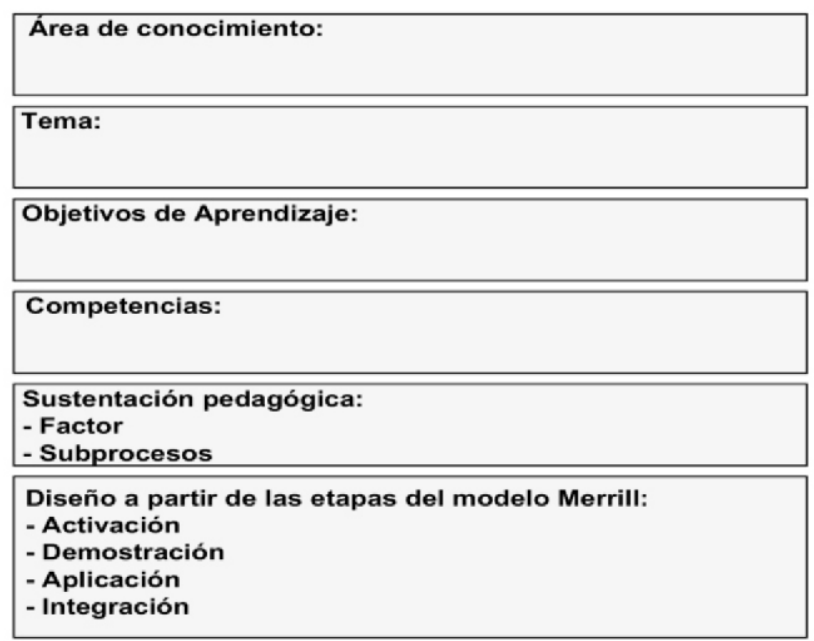

Figura 10. Plantilla para el diseño instruccional de recursos digitales educativos.

La plantilla para el diseño instruccional de recursos digitales educativos, es un instrumento clave, que permite al profesor en formación y/o formado en TIC, preparar su clase, presentar la secuencia didáctica, con todas las actividades y los contenidos en perfecta correlación; reconocer la naturaleza de la tarea y garantizar que él mismo y sus estudiantes identifiquen, entiendan y comprendan el proceso mental implícito en el proceso de enseñanza y de aprendizaje, más el desarrollo de las competencias de acuerdo con su nivel.

Esta matriz también está disponible en el campus virtual. 


\section{EL APRENDIZAJE A TRAVÉS DEL JUEGO. NECESIDADES DE ESTUDIANTES SORDOS}

Es importante partir de dos conceptos claves: el juego y las necesidades de estudiantes sordos.

Además de formar parte de una comunidad lingüística minoritaria, cuyo factor esencial es la lengua de señas, se caracterizan por poseer modos de funcionamiento socioculturales y cognoscitivos diferentes a los de los oyentes: se autoidentifican en normas de comportamiento que rigen sus interacciones sociales e interpersonales.

Por ejemplo, el contacto ocular, las reglas que gobiernan el contacto y los roces físicos, el uso de expresiones faciales, la gesticulación, hechos que conducen a unas formas de relación y convivencia que difieren de las de los oyentes.

La cultura sorda se caracteriza por ser eminentemente visual, es decir, los sordos crean convenciones que derivan de la significación que tienen la visión y el espacio para ellos; se trata de una actitud, de una experiencia de vida diferente (INSOR 2006).

El equipo de investigadores ha considerado, los videojuegos y sus dispositivos específicos, útiles para mejorar el proceso de aprendizaje, porque son muy atractivos para los estudiantes, aumentan sin presión ni estrés, la posibilidad de desarrollo de sus habilidades cognitivas; el tiempo dedicado al autoaprendizaje, la motivación hacia el aprendizaje, sus niveles concentración y de atención mientras están trabajando y jugando (Kulik 1994).

Por otro lado, se destaca la importancia de incorporar en los programas educativos, herramientas pertenecientes al contexto histórico-tecnológico contemporáneo, en apoyo a la adquisición de las competencias necesarias para enfrentar adecuadamente el futuro (Hepp 1999).
Reafirmando, los videojuegos son estrategias metodológicas que favorecen el aprendizaje significativo y constructivo en entornos digitales lúdicos, motivadores para los alumnos; deben constituirse en recurso didáctico por su potencial educativo; en este sentido, se les da preferencia para que a través de la práctica, desarrollen la capacidad para solucionar problemas: vídeojuegos estratégicos, que coadyuven con la mejora en la toma de decisiones, la administración de recursos disponibles y la planificación de habilidades puestas en juego.

También los juegos de roles que recreen su vida real, asumiendo otros con funciones determinadas en interacción con el contexto.

Se resalta en este proyecto, la importancia de la autorregulación, como característica importante de los videojuegos diseñados, como sistema, como el conjunto de mecanismos que adaptan el juego y sus contenidos al nivel de dominio mostrado por el niño, haciendo un registro en estadígrafos, en ellos se puede leer el desempeño del niño, en cuanto a habilidades para jugar el videojuego, como a nivel de conocimientos y dominio de los contenidos educativos. Así, el vídeo juego es un mediador de aprendizajes y promotor de la zona de desarrollo próximo (Vigotsky, 1978).

Estos aspectos son de alta importancia en esta investigación, más el hecho de que se comercializan un sinnúmero de videojuegos para diversión, en los cuales se evidencian debilidades relacionadas con el componente pedagógico, más aún, en el desarrollo de aquellos que son de tipo educativo para niños sordos.

En el mercado virtual se encuentran aplicativos libres y material multimedia para la enseñanza con niños sordos, sin embargo, es muy restringido el material de videojuegos educativos, que potencien el desarrollo de inteligencia contextualizada en ellos.

Por lo anterior, se propone el uso de videojuegos para mejorar el aprendizaje de los niños sordos. Así, en su 
diseño, se incorporaron componentes pedagógicos, visibles en las actividades didácticas para aprender en el área de ciencias naturales y sociales.

Se escogió esta temática teniendo en cuenta las necesidades de esta población más los intereses determinados en los estándares del Ministerio de Educación de Colombia (2013), donde se propone formar científicos, desarrollando las competencias necesarias para la formación en ciencias naturales y ciencias sociales; a partir de la observación y la interacción con el entorno, la recolección de información y la discusión con otros, hasta llegar a la conceptualización, la abstracción y la utilización de modelos explicativos y predictivos de los fenómenos observables y no observables del universo.

Alrededor del mundo, los educadores muestran su experiencia y buenas prácticas en el uso de los videojuegos para influenciar positivamente el comportamiento y compromiso de los estudiantes con su formación, desde tempranas etapas de su crecimiento. Meyer (2015) identifica cuatro formas innovadoras para el uso de los videojuegos en las aulas de clase que se mencionan a continuación.

- Aprendizaje basado en proyectos: busca introducir los videojuegos como un elemento pedagógico, que no necesariamente tiene una relación con las actividades curriculares, pero que permite a través de una temática específica, desarrollar competencias relacionadas con el área deseada gracias a la interacción controlada con los elementos audiovisuales del juego.

- Aprendizaje mixto (b-learning): gran parte del aprendizaje no se da al interior de las aulas, por lo que la utilización de videojuegos específicos con instrucciones del docente permite que el estudiante en sus ratos libres se divierta y a la vez aprenda.
- Juegos online: algunos juegos en línea desarrollan habilidades lectoescritoras en los estudiantes por la interacción con los demás jugadores conectados. Además el docente puede desarrollar actividades de comprensión de lectura con las historias y manuales de uso de estos juegos.

La estrategia de videojuegos se ha aplicado para resolver diferentes necesidades educativas de población sorda. Se requiere entonces de otros contextos de aprendizaje como el uso de un videojuego que bajo orientaciones pedagógicas permitan el desarrollo cognitivo de los niños sordos y que faciliten y mejoren el proceso de aprendizaje de los niños sordos.

Aunque ya existen varios videojuegos realizados para niños sordos (Weaver et al. 2010), que describen la evidente mejora de la capacidad de adquisición de la lengua de señas americana, este estudio se desarrolló para cuantificar el impacto del juego en el desarrollo lenguaje utilizando doce participantes.

También el trabajo de Henderson et al.(2005) propone una herramienta interactiva para el desarrollo de la lengua de señas americana con el apoyo de un tutor. En el contexto colombiano, existen trabajos como el de Jiménez (2007), quien considera necesario incorporar el modelo lingüístico en la ejecución de los videojuegos para incrementar la comprensión del tema.

El videojuego fortalece la apropiación de la lengua de señas colombiana y de la lengua escrita, facilita el aprendizaje de contenidos en ciencias naturales y sociales predeterminados en los estándares del Ministerio de Educación. Este recurso muestra cómo el modelo de aprendizaje, integra el uso de los sentidos para que los niños sordos desarrollen su capacidad para experimentar, imaginar y describir.

- Metodología para el diseño y la producción de videojuegos educativos: para esto, se definió el 
concepto, describiendo la idea central, los objetivos, los personajes, los entornos, las interfaces y las reglas y retos que deberá superar el jugador, constituyéndose así la filosofía del vídeo juego.

Al unísono, durante la fase de planeación se integró un grupo interdisciplinario constituido por expertos como pedagogos con experiencia en lengua de señas colombiana, diseñadores instruccionales, diseñadores y animadores digitales y programadores, con el acompañamiento de representantes de la comunidad sorda, todo para integrar el videojuego resultante en el motor UNITY3D.

Con relación a la disciplina de videojuegos, los propuestos se categorizarán como juegos serios (serious games), cuyo objetivo principal no es la diversión. Estos se han usado en distintas disciplinas de la educación dirigidas a jóvenes y adultos para iniciar, mejorar y facilitar el aprendizaje.

Se deben considerar cuatro componentes estructurales en un juego serio: objetivos, reglas, retos e interacción (Grau et al. 2015). El uso de estos componentes, determinaran el orden, los derechos y responsabilidades de los jugadores. Además, permitirán al jugador enfrentarse a problemas para los cuales tendrá que buscar soluciones.

Con respecto a la interacción en un juego serio, esta surge de la propia mecánica y dinámica del juego, dando lugar a las vivencias del jugador.

\subsection{Fases para la producción de un videojuego}

El juego es una actividad libre que se realiza sin una obligación externa, que incentivará al alumno a experimentar, a probar múltiples soluciones, descubrir la información y nuevos conocimientos sin temor a equivocarse.

En un juego, al ser versátil e interactivo, se pueden fijar distintos objetivos de aprendizaje. Estos aspectos se consignan en la plantilla para el diseño de recursos digitales educativos, cumpliendo con lo planteado por Acerenza et al. (2009), las tres fases para la producción de un videojuego: definición del concepto, planificación y elaboración.

\subsubsection{Definición del concepto}

En esta fase se clarifican aspectos de contextualización tales como el público objetivo, el modelo de negocio, las características de los personajes, la historia y los aspectos técnicos (lenguajes y herramientas para el desarrollo).

Nuestro principal interés son los contenidos y actividades de carácter pedagógico que atiendan los estándares del MEN, como se menciona a continuación.

(a) Videojuego en el área de conocimiento en Ciencias Sociales.

Tema: señales de tránsito.

Objetivo: potenciar la ubicación en el entorno físico y representación utilizando referentes espaciales.

Público objetivo: niños de Grados: $1^{\circ}$ y $3^{\circ}$.

Competencias: establecer relaciones entre los espacios físicos que ocupa el niño y sus representaciones, identificar situaciones cotidianas que indican cumplimiento de las funciones de algunas organizaciones sociales del entorno; identificar normas que rigen algunas comunidades a las que pertenece y explicar su utilidad; reconocer normas construidas socialmente y distinguir aquellas en las que se puede participar. También participar en la construcción de normas para la convivencia en los grupos sociales y políticos a las que pertenece y valorar aspectos de las organizaciones sociales y políticas del entorno que promueven el desarrollo individual y comunitario.

(b) Videojuego en el área de Conocimiento en Ciencias Naturales. 
Tema: la vida de los dinosaurios.

Objetivo: conocer sobre el origen del ser vivo y su comportamiento.

Público objetivo: niños de Grados: $6^{\circ}, 7^{\circ}$ y $8^{\circ}$.

Competencias: reconocer el origen del universo y de la vida a partir de varias teorías, establecer relaciones entre el clima en las diferentes eras geológicas y las adaptaciones de los seres vivos; y comparar diferentes teorías sobre el origen de las especies.

El modelo instruccional de Merrill (2007) para el desarrollo de Contenido Digital Educativo, toma como centro del ejercicio pedagógico el "problema", desarrolla aprendizaje significativo, demuestra la utilidad del conocimiento y las posibilidades de aplicación e integración con el entorno de quien aprende, que en este caso serían los niños sordos.

Merrill señala cuatro estadios para abordar el problema que aparecen a continuación.

- Activación: es decir, identificar en el estudiante los pre-saberes. Al inicio de cada videojuego se le contará una historia corta sobre el personaje principal, que en el caso del videojuego "La Vida de los Dinosaurios", será un dinosaurio, y para el caso del videojuego de las señales de tránsito, el jugador será un pizzero. En ambos casos se hará un recorrido por el escenario para que el niño jugador reconozca e identifique el contexto en que se encuentra.

- Demostración: se le indican al estudiante las ventajas del nuevo conocimiento y se le dará una explicación de cómo deberá jugar.

En el caso del videojuego de "La Vida de los Dinosaurios", el jugador tiene estas instrucciones: el jugador no puede salirse del rango del juego; si el jugador llegase a caer en el agua y este no es acuático, automáticamente perderá; por cada logro conseguido, se otorgarán puntos; el jugador debe alimentarse de los otros animales y debe tomar agua para sobrevivir de lo contrario este perderá.

En el videojuego sobre las señales de tránsito, el jugador deberá realizar cinco entregas, hacer las entregas en el tiempo que se le indique; prestar atención para que no infrinja ninguna norma de tránsito, de lo contrario pondrá en peligro el puesto que pretende alcanzar.

- Aplicación: el aprendiz aplica el nuevo conocimiento adquirido en diferentes situaciones. Se diseñarán pruebas que el niño deberá superar para ganar puntos, por ejemplo, en el caso del videojuego "La vida de los Dinosaurios", un dinosaurio podrá sentir hambre o sed, por lo que el jugador deberá buscar para sobrevivir.

En el caso del videojuego sobre señales de tránsito, el pizzero, en caso que se le esté agotando el tiempo para las entregas, deberá tomar las rutas más cortas y menos transitadas, siempre y cuando reconozca las reglas de tránsito construidas socialmente. De esa manera logrará obtener los puntos necesarios para ganar. Además contará con ayudas como advertencias, notificaciones de fallos y retroalimentación.

- Integración: en el videojuego "Señales de Tránsito", el niño se concientizará sobre sobre las buenas prácticas al momento de transitar en el espacio público.

\subsubsection{Planificación}

En esta fase se pueden realizar dos actividades importantes, la primera es la planificación administrativa en la que se define el cronograma, el equipo de trabajo y el presupuesto.

Conviene subrayar que el proceso de producción del videojuego exige la constitución de un equipo interdisciplinario. Al interior de este, debe haber un experto pedagógico y un experto en población sorda. El conocimiento técnico será aportado por los estudiantes de programas de informática, quienes 
desempeñarán los roles de experto tecnológico (técnico líder) y desarrollador de videojuego.

Además, se contará con personal de soporte en animación digital y diseño de interfaz gráfica.

La segunda actividad concierne a la especificación del videojuego, es decir, a la definición de las características funcionales (desde el punto de vista del usuario final), y las características no funcionales (cualidad que el videojuego debe presentar).

\subsubsection{Elaboración}

En esta fase se realizan las tareas específicas para cada una de las características funcionales y no funcionales del videojuego.

Se plantea una tarea, luego se ejecuta y finalmente se le hace seguimiento para validar su concreción.

En lo que respecta al videojuego propuesto, cada tarea puede involucrar actividades de diseño y de modelación de escenarios, personales, objetos, animaciones y sonidos.

Por otro lado, esta instancia de elaboración implica la renderización de los recursos digitales y su integración en Unity 3D (2013), el cual es el seleccionado para el desarrollo del primer prototipo del videojuego, ya que es un potente motor de renderizado, totalmente integrado de herramientas intuitivas y flujos de trabajo o rápidos para crear contenido 3D interactivo. Soporta además el despliegue de múltiples plataformas incluyendo teléfonos móviles webs, escritorios y consolas.

En la Fig. 11, se muestra el diseño de los escenarios principales, una ciudad y un bosque.

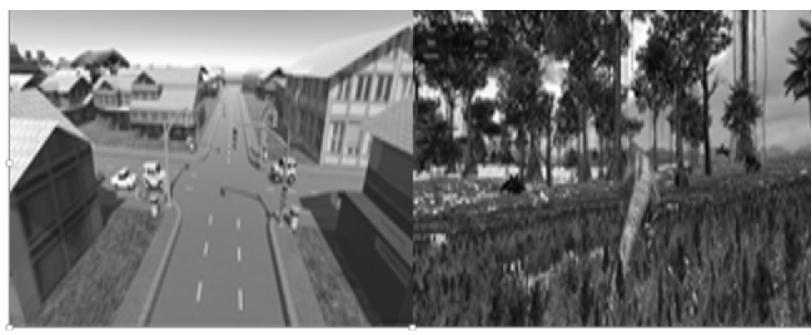

Figura 11. Escenarios de videojuegos.

La Figura 12 muestra objetos modelados que se integraron a los escenarios para brindarle una apariencia real.

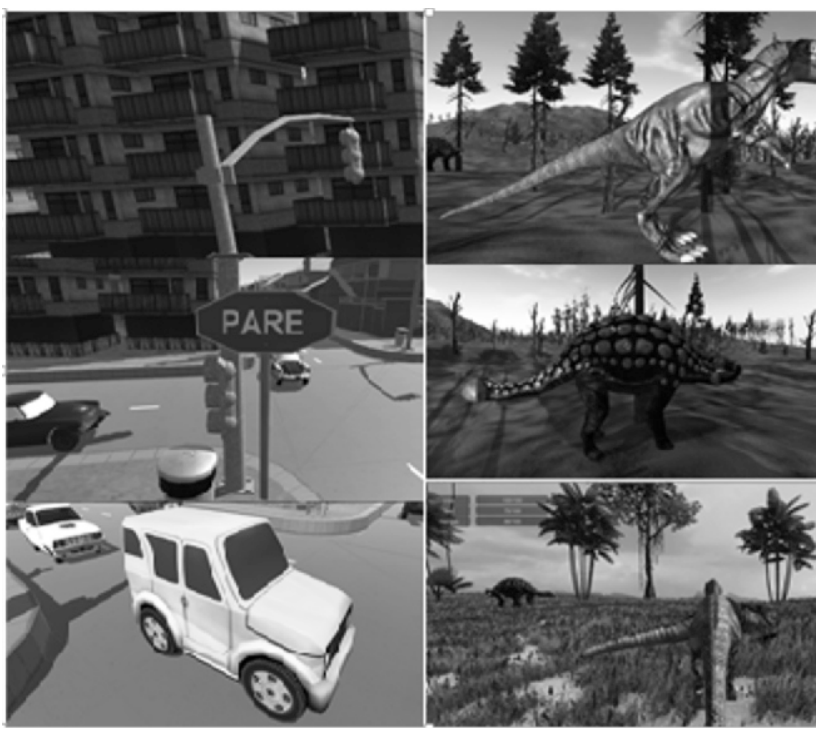

Figura 12. Objetos y elementos modelados para escenario de los videojuegos

\section{CONCLUSIONES}

(a) La línea base reveló la necesidad de formación en TIC de los profesores de las instituciones educativas, además de su deseo de vincularse a proyectos de investigación que les dejen conocimientos y les den herramientas para la inclusión de población diversa. 
(b) Los profesores que tienen en sus escenarios de enseñanza población sorda y otras condiciones de discapacidad, reconocen el potencial didáctico de los recursos y contenidos digitales educativos, los valoran por el aumento de la motivación, del interés y del grado de atención y concentración que se logra con estudiantes cuyo desempeño no alcanza la media esperada, tanto de regulares como de sordos.

(c) El Instituto Nacional de Sordos se vincula como entidad de apoyo a las actividades que se realizan en campo, a hacer acompañamiento durante la fase de pilotaje de los cursos para diseño y producción de contenidos digitales educativos para sordos en diversas áreas de conocimiento.

(d) El campus virtual se visiona como un gestor de contenidos, concentrador de actividades en torno al mejoramiento de las competencias profesionales del docente, como también del desempeño de los estudiantes en condición de sordera como los regulares; también como un generador de comunidades de aprendizaje y además, se podrá modelar las relaciones entre los participantes de las comunidades a través del modelado por redes

(e) El trabajo interdisciplinario ha permitido integrar diferentes campos, de la pedagogía y la ingeniería, de entidades oficiales y privadas, la comunidad académica y educativa, administrativos y docentes de las instituciones; e incluir, hablando de diversidad, a la comunidad sorda y oyente.

(f) Adicionalmente, se han podido establecer otros parámetros de trabajo e investigación que bien vale considerar para su profundización en lo actual y a futuro, como los estilos cognitivos de los estudiantes sordos, el conocimiento y manejo de TIC por parte de docentes y alumnos, los planes de estudio y las adecuaciones para favorecer los procesos de enseñanza y aprendizaje; la infraestructura tecnológica de las instituciones, las teorías pedagógicas y metodológicas vigentes en las instituciones con estudiantes sordos y en general, con contextos inclusivos.

(g) Así como los aspectos administrativos, recursos educativos y políticas que allí se encuentran.

(h) El modelo ha permitido también adentrarse en principios políticos, educativos y filosóficos para trabajar con la tecnología en el mejoramiento de la enseñanza y el aprendizaje.

(i) Se ha propiciado simultáneamente, la gestión de procesos, conocimientos y la creación de comunidades de aprendizaje; así como el profundizar en principios valiosos como el de la incertidumbre, los que dan la base al Diseño Universal de Aprendizaje, los modelos y las competencias con respecto a TIC y los lineamientos desde el Ministerio Nacional de Educación para la construcción de una sociedad en paz, más equitativa y educada.

(j) Con respecto al diseño de los videojuegos educativos se consideraron aspectos esenciales como ambientes o escenarios, mecanismos, motivación y sociabilidad.

(k) Los videojuegos educativos se apoyan fuertemente en la narrativa, la integración de los factores seleccionados permiten aumentar la sensación de realidad de los mundos virtuales y las interacciones con el mismo, gracias a la integración de la tecnología $3 \mathrm{D}$ y de los efectos audiovisuales es posible captar la atención del jugador, e indudablemente, estimulan en gran medida a los estudiantes sordos dado que gran parte de la información del entorno, la captan mediante la visión y la parte kinestésica táctil.

(I) Con el diseño de los videojuegos se favorece el proceso de aprendizaje, pues son muy atractivos para todo público, en especial el infantil y juvenil; aumentan sus habilidades cognitivas, el tiempo dedicado a aprender, su motivación para el aprendizaje, su concentración y su atención mientras 


\section{.. H.'Ingenierías}

están trabajando y/o jugando.

(m) Es importante resaltar, que la tecnología no va sin un guía, y que la integración de los contenidos pedagógicos depende del conocimiento, objetivos y plan de estudios del docente, es él quien debe introducir los conceptos a trabajar en los videojuegos e ir enriqueciendo los mismos, acorde a lo que se vaya dando durante su desarrollo y aprovechando al máximo la motivación que éstos despierten, para fortalecer los aprendizajes de parte y parte.

Debido a la tendencia actual del uso de la multimedia y el diseño de cursos que se adaptan al estilo de aprendizaje del estudiante, este modelo pretende formar parte de un mundo más accesible para todos en términos de formación e información, pero es el orientador o docente quien hace que sea utilizado de la mejor manera, basado en una pedagogía amena, interactiva, lúdica, de verdaderos aprendizajes y retos y definitivamente incluyente.

\section{REFERENCIAS}

Acerenza, N., Mesa, G., Viera, A., Fernández, E., Laurenzo, T. y Vallespir, D. (2009). Una metodología para el desarrollo de video juegos. Presentado en 380 JAllO- Simposio Argentino de Ingeniería de Software, pp. 171-176.

Barcena, F. (1994). La práctica reflexiva en educación / Reflective practice in education, 1.a ed. Madrid: Universidad Complutense de Madrid.

García, F. (2000). Los modelos didácticos como instrumento de análisis y de intervención en la realidad educativa. Rev. Biblio $3 \mathrm{~W}$ Rev. Bibliográfica Geogr. Cienc. Soc., 5(207), 12.

Grau, S., Reig, R., Puig, A., López, M. y Rodríguez, I. (2015). Games4Learning: Cómo integrar juegos serios en itinerarios de aprendizaje personalizados?. Games4Learning Integrate Serious Games Pers. Learn. Itiner., (1), 1275-1280.

Heisenberg, W. (1985). Gesammelte Werke. Series C: Philosophical and Popular Writtings, 3. München: Piper, 1985.

Henderson, V., Lee, S., Brashear, H., Hamilton, H., Starner, T. y Hamilton, S. (2005). Development of an American Sign Language Game For Deaf Children. Dev. Am. Sign Lang. Game Deaf Child.

Hepp, P. (1999). La red Enlaces del Ministerio de Educación de Chile. Cienc. Al Día, 2(3).

INSOR. (2006). Educación Bilingüe para sordos. Bogotá: Imprenta Nacional.

Jiménez, L. (2007). Juegos para niños sordos de 5 a 7 años. Chía.

Kulik, J. (1994). Technology Assesment in Education and Training. Technology assesment in education and 
training. New York: Lawrence Erlbaum Associates, Inc. Kriegel, U. (2009). Teorías de la conciencia. Prax. Filosófica, (29), 179-187.

Merrill, M. D. (2007). First principles of instruction: A synthesis. In R. A. Reiser \& J. V. Dempsey (Eds.), Trends and issues in instructional design and technology (2nd ed., pp. 62-71). Upper Saddle River, NJ: Merrill/Prentice-Hall.

Meyer, L. (2015). 4 Innovative Ways to Teach With Video Games. TH E J., 42,(5), 20-24.

Ministerio de Educación Nacional. (2013). Competencias TIC para el desarrollo profesional docente, 1.a ed. Bogotá: Imprenta Nacional, 2013.

Ministerio de Educación Nacional. (2004). Estándares Básicos en competencias en ciencias naturales. Recuperado de

http://www.mineducacion.gov.co/cvn/1665/articles81033_archivo_pdf.pdf (2004)

Morin, E. (1993). El Método. Naturaleza de la naturaleza. Madrid: Cátedra.

Nesbit, J., Belfer, K. y Leacock, T. (2003). Instrumento para a Avaliação de Objectos de Aprendizagem (LORI). UNESCO. (2006). La Educación Inclusiva: el camino hacia el futuro. Presentado en Cuadragésima octava reunión, Ginebra. pp. 37.

Unity3D. (2013). Documentation, ScriptReference.

Unity Scripting Reference. Unity3D. Recuperado de http://unity3d.com/learn/documentation

Universidad Internacional de Andalucía. (2008). La escuela de la diversidad: educación inclusiva, construyendo una escuela sin exclusiones". El camino de la inclusión educativa en Punta Hacienda. Andalucía.
Vigotsky, L. (1978). El desarrollo de los procesos psicológicos superiores, 1.a ed. Barcelona: Editorial Crítica.

Vitoriano, B. (2008). Teoría de la decisión: decisión con incertidumbre, decisión multicriterio y teoría de juegos. Madrid: Universidad Complutense de Madrid. Weaver, K., Hamilton, H., Zafrulla, Z., Brashear, H., Starner, T., Presti, P. y Bruckman, A. (2010). Improving Then Language Ability Of Deaf Signing Children Through An Interactive American Sign Language Based Video Game. ICLS, (2).

Wenger, E. (2001) Comunidades de práctica. Aprendizaje, significado e identidad. Barcelona: Paidós. 